\title{
Social Networks, Community Engagement, and Cognitive Impairment among Community-Dwelling Chinese Older Adults
}

\author{
Jinlei Li Zijuan Wang Zhiwei Lian Zhikai Zhu Yuanli Liu \\ School of Public Health, Peking Union Medical College, Beijing, China
}

\section{Keywords}

Cognitive impairment $\cdot$ Community engagement $\cdot$ Social networks $\cdot$ Aging

\begin{abstract}
Aims: To examine the association of social networks and community engagement with cognitive impairment among community-dwelling Chinese older adults. Methods: From November 2017 to May 2018, we selected 1,115 elderly individuals from 3 Chinese communities (Beijing, Hefei, and Lanzhou) using a random-cluster sampling method, and recorded data on demographics, social network characteristics, community activities, and cognitive function. The odds ratios (ORs) of these associations were adjusted for potential confounders in logistic regression models. Results: The prevalence of cognitive impairment was $25.7 \%(n=287)$. An adequate social network (OR $0.55 ; 95 \%$ confidence interval $[\mathrm{Cl}] 0.33-0.91$ ) and enough social support from friends (OR $0.43 ; 95 \% \mathrm{Cl} 0.29-0.62$ ) were negatively associated with cognitive impairment. Family support was not significantly associated with cognitive impairment (OR 0.64; 95\% Cl 0.34-1.21). Taking part in elderly group travel, communication with others using WeChat, and community activities such as Tai Chi and walking together were negatively associated with cognitive impairment. Conclusion: Social network characteristics and community engagement were found to be related to cognitive function among community-dwelling Chinese elderly adults.

(C) 2019 The Author(s)

Published by S. Karger AG, Basel
\end{abstract}

\section{Introduction}

Cognitive impairment is an important public health problem. With the population aging, more people will begin to have age-related disorders, including cognitive impairment [1]. In China, $>10 \%$ of older individuals suffer from cognitive impairment, ranging from mild deficits to dementia $[2,3]$. Therefore, studying the determinants of cognitive function to 
Li et al.: Social Networks, Community Engagement, and Cognitive Impairment among Community-Dwelling Chinese Older Adults

reduce the incidence of cognitive impairment is becoming an increasingly important area of research [1].

Much of the research on dementia and cognitive impairment focuses on the identification of biomarkers decades before the threshold for clinical diagnosis is met [4, 5]. Most biomarker studies have concentrated on measures of $\beta$-amyloid detected by PET imaging or measured in the cerebrospinal fluid (CSF). However, nearly $75 \%$ of cognitively normal individuals aged 70-79 years are free of significant amyloid on PET imaging [6]. Along with this biomarker research, numerous studies have reported that modifiable behaviors such as lifestyle factors, social contacts, and community activities are also related to an increased risk for cognitive impairment, although the results have shown varying degrees and strengths of these relationships [4, 7-11].

Emerging evidence suggests that social support networks may have a positive influence on cognition and a protective effect against the development of cognitive impairment among older adults $[10,12,13]$. A cohort study found that after controlling for a variety of risk factors, those with no social ties had a $>2$-fold increased risk of being cognitively impaired compared with those who had 5 or 6 social ties [14]. The Honolulu-Asia Aging Study showed that little social engagement in later life was associated with the risk of dementia, and that levels of later-life social engagement may have already been modified by the dementing process and may be associated with prodromal dementia [15]. More recent studies have suggested that larger social networks have a protective influence on cognitive function among elderly individuals $[1,8,10]$.

We expanded on these previous studies by recruiting a community-dwelling elderly population sample from 3 cities in China, and then applied widely used and validated measures of social network characteristics and cognition. We aimed to investigate the associations between social networks, community engagement, and cognitive impairment in Chinese elderly adults.

\section{Methods}

\section{Participants}

This study is an observational cross-sectional study. Participants were selected from 3 cities in China: Beijing (North China), Hefei (Central China), and Lanzhou (Northwest China). We selected 1 aged community from each city. From November 2017 to May 2018, approximately 400 residents aged $>60$ years were randomly selected from each community. When a selected individual refused or was unavailable, a replacement was selected from the same community. We excluded those with severe mental disorder and hearing impairment, and the final sample size was 1,115 (with 85 incomplete questionnaires [7.1\%]). Demographic and lifestyle factors were obtained from questionnaires conducted by trained community nurses. Age was divided into 3 groups: 60-69 years, 70-79 years, and $>80$ years. Marital status included: single, married, widowed, divorced/separated. Education levels were classified into: primary school and under, middle school, and college and over. Living status was recorded as living alone, with a spouse and/or one's children, and with others.

\section{Cognition Measurement}

The Mini-Mental State Examination (MMSE) screening test was administered to participants by trained nurses. The MMSE is a measure of general cognitive function that measures orientation to time and place, attention and calculation, and language and memory [16]. Summed scores from the MMSE ranged from 0 to 30, where higher values 
denoted better cognitive functioning. Cognitive impairment was defined as having an MMSE score $<24$.

\section{Assessment of Social Networks}

Our social network measure was the abbreviated Lubben Social Network Scale (LSNS-6). This measure contains 6 questions: 3 key questions evaluate the sizes of 3 different aspects of social networks that are attributable to family ties and a parallel set attributable to friendship ties. The LSNS- 6 assesses the size of the respondent's active social network (i.e., relatives or friends seen or heard from $\geq 1$ times/month), perceived support network (i.e., relatives or friends who could be called on for help), and perceived confidant network (i.e., relatives or friends to whom the respondent could talk about private matters) [10]. Each LSNS-6 question is scored on a scale of 0-5. The total score is an equally weighted sum of these 6 questions, with scores ranging from 0 to 30 . We also summed the subscale scores for family and friends, with results ranging from 0 to 15 [10]. Higher scores indicated larger social networks. The validated LSNS-6 has been widely used and a cutoff of 12 has been established to provide the best overall sensitivity [17, 18].

Community engagement was measured by the frequency $(0=$ never, $1=$ sometimes, $2=$ often) with which a subject engaged in 5 popular community activities: public square dancing, elderly group travel, university for seniors, communication using WeChat ( a mobile app for communication), and activities such as Tai Chi (a gentle form of exercise for the elderly) and walking together. Persons who self-reported "sometimes" and "often" were classified as participants, while those who self-reported "never" were classified as nonparticipants in this study.

\section{Analyses}

Descriptive statistics were generated for demographic variables including age, sex, education, marital status, and living arrangement. We first performed a univariate logistic regression analysis, followed by a model adjusted for age, sex, and education; the model further accounted for marital status and living arrangement, which may affect an individual's social network or cognitive function. We estimated odds ratios (ORs) for cognitive impairment in those who scored high on the LSNS-6 total scale (with scores of $0-11$, below the cutoff, as the reference) and those scoring higher on the LSNS- 6 family and friends subscales (with scores of $0-5$, below the subscale cutoffs, as the reference). The associations between each social activity and cognitive impairment were determined, and 95\% confidence intervals (CIs) were calculated for the OR estimates. All analyses were performed using STATA v15.0.

\section{Results}

A total of 1,115 participants (aged $\geq 60$ years; range 60-99 years) completed the cognition test and social support questionnaire. After cognitive function screening (median MMSE score: 24; p25-p75: 19-28), 287 participants (25.7\%) were considered to be cognitively impaired. The crude OR, 95\% CI, and proportions of various factors are shown in Table 1 . As expected, advanced age was strongly associated with cognitive impairment. We divided participants into 3 age groups; not surprisingly, the cognitive impairment in the groups aged 70-79 years and $>80$ years was 1.33-fold and 1.69-fold higher than in the group aged 60-69 years. Female participants showed poorer cognitive function than males. This finding was consistent with research studies in China that found women were signifi- 
Li et al.: Social Networks, Community Engagement, and Cognitive Impairment among Community-Dwelling Chinese Older Adults

Table 1. Demographic characteristics of participants

\begin{tabular}{|c|c|c|c|c|c|}
\hline & \multirow[t]{2}{*}{$N$} & \multicolumn{2}{|c|}{$\begin{array}{l}\text { Patients with cognitive } \\
\text { impairment }\end{array}$} & \multirow[t]{2}{*}{ Crude OR } & \multirow[t]{2}{*}{$95 \% \mathrm{CI}$} \\
\hline & & $n$ & $\%$ & & \\
\hline Number of patients & 1,115 & 287 & 25.7 & & \\
\hline \multicolumn{6}{|l|}{ Sex } \\
\hline Male & 435 & 74 & 17.0 & 1 & \\
\hline Female & 680 & 213 & 31.3 & 2.25 & $1.65-2.99$ \\
\hline \multicolumn{6}{|l|}{ Age group } \\
\hline 60-69 years & 471 & 99 & 21.0 & 1 & \\
\hline $70-79$ years & 354 & 94 & 26.6 & 1.33 & $0.91-1.94$ \\
\hline$\geq 80$ years & 290 & 94 & 32.4 & 1.69 & $1.11-2.57$ \\
\hline \multicolumn{6}{|l|}{ Education } \\
\hline Primary school and under & 535 & 231 & 43.2 & 1 & \\
\hline Middle school & 419 & 45 & 10.7 & 0.18 & $0.12-0.26$ \\
\hline College and over & 132 & 11 & 8.3 & 0.13 & $0.07-0.26$ \\
\hline \multicolumn{6}{|l|}{ Marital status } \\
\hline Married & 705 & 155 & 22.0 & 1 & \\
\hline Widowed & 357 & 121 & 33.9 & 1.82 & $1.37-2.41$ \\
\hline Single & 16 & 6 & 37.5 & 2.13 & $0.76-5.95$ \\
\hline Divorced or separated & 37 & 5 & 13.5 & 0.55 & $0.21-1.45$ \\
\hline \multicolumn{6}{|l|}{ Living arrangement } \\
\hline Alone & 240 & 51 & 25.8 & 1 & \\
\hline With a spouse or children & 852 & 52 & 22.8 & 1.37 & $0.97-1.93$ \\
\hline With others & 23 & 6 & 26.1 & 1.31 & $0.49-3.49$ \\
\hline
\end{tabular}

cantly disadvantaged in cognitive functioning in old age [19]. The singles group had significantly higher risks than the groups with married people. A higher education level was associated with a reduced risk of cognitive impairment. Living arrangements were not significantly related to cognitive impairment among the elderly individuals in these 3 urban communities.

Social network scores were associated with cognitive impairment. Table 2 shows the crude and adjusted ORs for cognitive impairment according to characteristics of the social network and social support. We calculated the crude OR for cognitive impairment on the LSNS-6 total to be 0.46 (95\% CI 0.29-0.71). With the lower subscale cutoff score (0-5) as the reference, the crude OR for cognitive impairment was 0.70 (95\% CI 0.40-1.21) for the family subscale and 0.32 (95\% CI 0.23-0.45) for the friends subscale. Controlling for age, sex, education, marital status, and living arrangement, with the lower LSNS-6 cutoff score (0-11) as the reference, the adjusted OR for cognitive impairment on the LSNS- 6 total was 0.55 (95\% CI 0.33-0. 91). With the lower subscale cutoff score (0-5) as the reference, the adjusted OR for cognitive impairment was 0.64 (95\% CI 0.34-1.21) for the family subscale and 0.43 (95\% CI 0.29-0.62) for the friends subscale.

Table 3 indicates the association of the 5 community activities with cognitive impairment. Public square dancing, elderly group travel, university for seniors, communication using WeChat, and activities such as Tai Chi and walking together were all associated with a lower risk of cognitive impairment in the univariate model. After adjustment for age, sex, education level, marital status, and living arrangement, taking part in elderly group travel, communication with others using WeChat, and activities such as Tai Chi were found to reduce the risk of cognitive impairment (OR 0.39 [95\% CI 0.25-0.60], OR 0.23 [95\% CI 0.13-1.41], and OR 0.63 [95\% CI 0.44-0.91], respectively). 

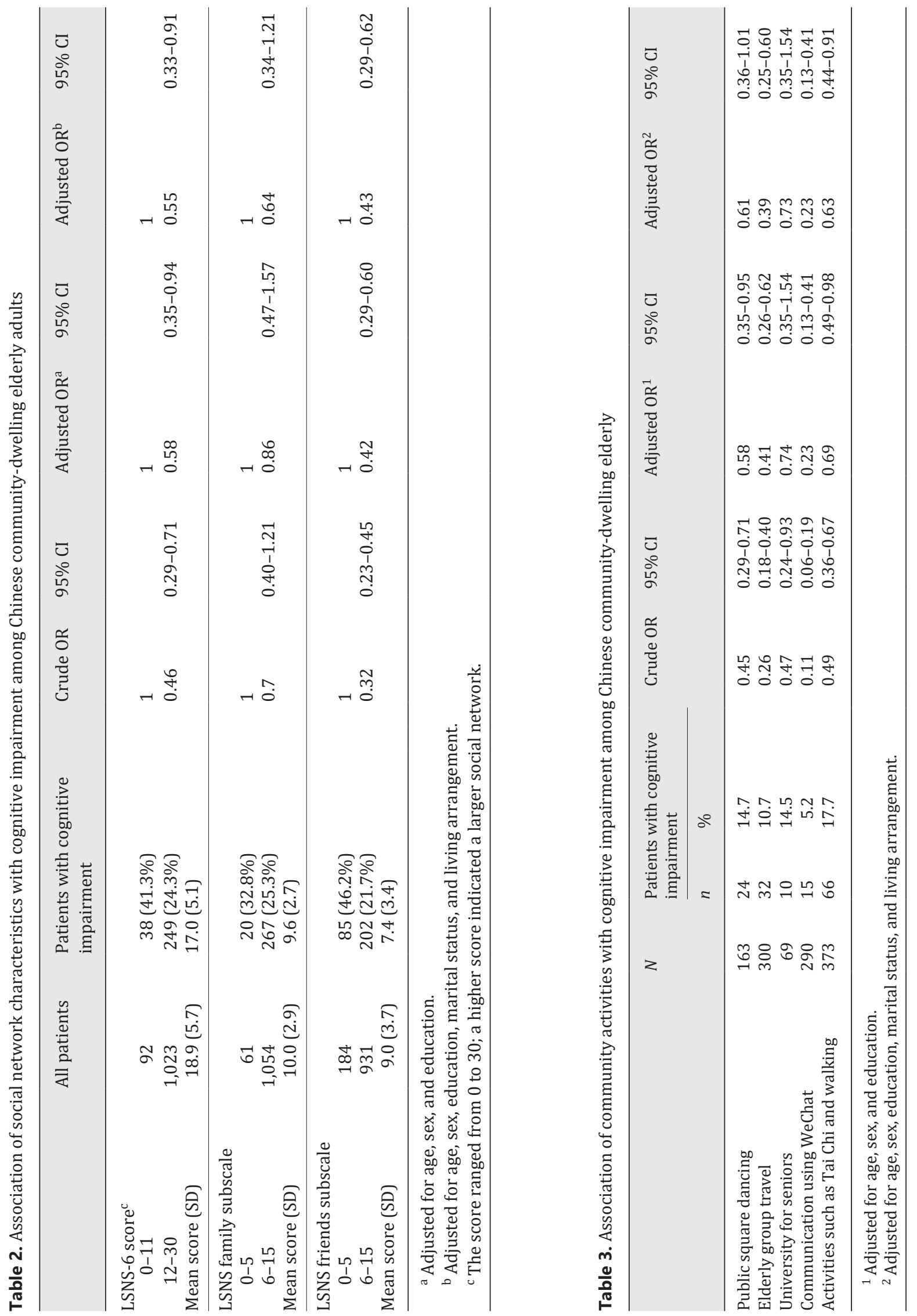


\section{Discussion}

We enrolled 1,115 residents from 3 urban communities to assess the association of social network characteristics and cognitive impairment. Social network characteristics were associated with differential risks of cognitive impairment. Persons with adequate social support from friends had better cognitive function, while social support from families was not significantly associated with cognitive impairment in the communities in all 3 cities. We further analyzed the effects of 5 community activities on cognitive impairment. After adjusting for age, sex, education, marital status, and living arrangement, elderly group travel, communication using WeChat, and activities such as Tai Chi and walking together were found to be negatively associated with cognitive impairment [20].

The associations in this work were based on a cross-sectional study, and reverse causation may exist. It is likely that at the beginning of cognitive impairment, people lose some social ties and stop engaging in social events and sports. However, our results are consistent with previous longitudinal studies in which a lack of social ties was associated with an increased risk of cognitive decline $[10,14,21]$. A cohort study found that larger social networks had a protective influence on cognitive function among elderly women in the USA [10]. Social networks were identified as a protective factor against cognitive impairment in Chinese older adults in the USA in the PINE study, while various dimensions of social networks were found to have different relationships with the global and particular domains of cognitive function [8]. The status of being single, having no close friends, and having a low level of social contact were associated with an increased risk of cognitive impairment in Chinese nonagenarians and centenarians in the PLAD (Longevity and Aging in Dujiangyan) project [1]. These findings suggested that social networks may facilitate access to health care and promote healthy behaviors, and thereby indirectly reduce or forestall brain pathology and other conditions that affect cognition [10]. Nevertheless, establishing a direct link between social networks and cognitive functioning and dementia is necessarily complex [22].

The concept of a social network has been defined in various ways in the academic literature [23]. Our study assessed social networks with a widely used questionnaire. The LSNS-6 assesses 2 aspects of social networks: families and friends. In this study, there was a significant association with the friends subscale but the association was not significant with the family subscale. This may indicate that, compared with family members, friends or neighbors play more important roles in the protection against cognitive impairment. Communications through WeChat could contribute to interactions between friends or neighbors among urban community residents. To date, effective interventions to help reduce the prevalence of cognitive impairment are limited. Social networks are an amenable influencing factor on

cognitive function, and an increase in social engagement is associated with a lower risk of cognitive impairment. As such, interventions tailoring social networks in elderly individuals may help delay age-related cognitive decline. Community participation may also help reduce cognitive decline [24, 25]. Community management could coordinate elderly group travel and activities such as Tai Chi for not only cognitive impairment protection but also other public health benefits in community-dwelling Chinese elderly adults [26, 27].

Our study had several limitations. First, it was a cross-sectional study and could not provide causal relationships between social network characteristics and cognitive impairment. Additional longitudinal studies are needed to validate the findings. Second, selection and recall biases of the participants posed an additional problem. Cognitive decline and early dementia may result in a reduction in interactions and social network support, or it can lead to a failure to recall and report offered support. Third, a residual confounding effect from unmeasured variables was possible and may have contributed to the observed associations. The presence of coexisting diseases and apolipoprotein E $[28,29]$ were not recorded in this 
Li et al.: Social Networks, Community Engagement, and Cognitive Impairment among

Community-Dwelling Chinese Older Adults

study; therefore, we could not adjust for hypertension, diabetes, stroke or genetic factors in the multivariable models. There are many kinds of community activities, but we only assessed 5 popular activities in this study. Furthermore, the MMSE is not a test of cognitive function, but rather a screening instrument. Further studies should use cognitive function tests and analyze other community activities. Lastly, it is possible that the associations may not be fully generalizable outside of this study because the sample comprised individuals from only 3 urban communities.

In summary, we showed that social network characteristics and community engagement were related to cognitive function among Chinese community-dwelling older individuals. We found that a social network with closer friends and greater community engagement, such as elderly group travel, communication with others using WeChat, and activities such as Tai Chi were associated with higher MMSE scores. Intervention studies examining the effect of improving social networks for the prevention of cognitive impairment are required in the future.

\section{Statement of Ethics}

The study protocols and participant consent forms were approved by the Chinese Academy Medical Sciences (X170315009); all participants provided written informed consent.

\section{Disclosure Statement}

The authors declare that they have no financial support or relationships that may pose conflicts of interest.

\section{Funding Source}

This work was supported by the Non-profit Central Research Institute Fund of Chinese Academy of Medical Sciences and the Natural Science Foundation of China (71661167004).

\section{References}

1 Wang B, He P, Dong B. Associations between social networks, social contacts, and cognitive function among Chinese nonagenarians/centenarians. Arch Gerontol Geriatr. 2015 May-Jun;60(3):522-7.

2 Chan KY, Wang W, Wu JJ, Liu L, Theodoratou E, Car J, et al.; Global Health Epidemiology Reference Group (GHERG). Epidemiology of Alzheimer's disease and other forms of dementia in China, 1990-2010: a systematic review and analysis. Lancet. 2013 Jun;381(9882):2016-23.

3 Nie H, Xu Y, Liu B, Zhang Y, Lei T, Hui X, et al. The prevalence of mild cognitive impairment about elderly population in China: a meta-analysis. Int J Geriatr Psychiatry. 2011 Jun;26(6):558-63.

4 Li J, Ogrodnik M, Kolachalama VB, Lin H, Au R. Assessment of the Mid-Life Demographic and Lifestyle Risk Factors of Dementia Using Data from the Framingham Heart Study Offspring Cohort. J Alzheimers Dis. 2018; 63(3):1119-27.

5 Li J, Ogrodnik M, Devine S, Auerbach S, Wolf PA, Au R. Practical risk score for 5-, 10-, and 20-year prediction of dementia in elderly persons: Framingham Heart Study. Alzheimers Dement. 2018 Jan;14(1):35-42.

6 Morris JC, Roe CM, Grant EA, Head D, Storandt M, Goate AM, et al. Pittsburgh compound B imaging and prediction of progression from cognitive normality to symptomatic Alzheimer disease. Arch Neurol. 2009 Dec; 66(12):1469-75.

7 Peters R, Peters J, Warner J, Beckett N, Bulpitt C. Alcohol, dementia and cognitive decline in the elderly: a systematic review. Age Ageing. 2008 Sep;37(5):505-12. 
Li et al.: Social Networks, Community Engagement, and Cognitive Impairment among Community-Dwelling Chinese Older Adults

8 Li M, Dong X. Is Social Network a Protective Factor for Cognitive Impairment in US Chinese Older Adults? Findings from the PINE Study. Gerontology. 2018;64(3):246-56.

9 Karp A, Paillard-Borg S, Wang HX, Silverstein M, Winblad B, Fratiglioni L. Mental, physical and social components in leisure activities equally contribute to decrease dementia risk. Dement Geriatr Cogn Disord. 2006; 21(2):65-73.

10 Crooks VC, Lubben J, Petitti DB, Little D, Chiu V. Social network, cognitive function, and dementia incidence among elderly women. Am J Public Health. 2008 Jul;98(7):1221-7.

11 Ge S, Wu B, Bailey DE, Dong X. Social Support, Social Strain, and Cognitive Function among CommunityDwelling U.S. Chinese Older Adults. J Gerontol A Biol Sci Med Sci. 2017 Jul 1;72(suppl_1):S16-21.

12 Green AF, Rebok G, Lyketsos CG. Influence of social network characteristics on cognition and functional status with aging. Int J Geriatr Psychiatry. 2008 Sep;23(9):972-8.

13 Holtzman RE, Rebok GW, Saczynski JS, Kouzis AC, Wilcox Doyle K, Eaton WW. Social network characteristics and cognition in middle-aged and older adults. J Gerontol B Psychol Sci Soc Sci. 2004 Nov;59(6):278-84.

14 Bassuk SS, Glass TA, Berkman LF. Social disengagement and incident cognitive decline in community-dwelling elderly persons. Ann Intern Med. 1999 Aug;131(3):165-73.

15 Saczynski JS, Pfeifer LA, Masaki K, Korf ES, Laurin D, White L, et al. The effect of social engagement on incident dementia: the Honolulu-Asia Aging Study. Am J Epidemiol. 2006 Mar;163(5):433-40.

16 Folstein MF, Folstein SE, McHugh PR. "Mini-mental state". A practical method for grading the cognitive state of patients for the clinician. J Psychiatr Res. 1975 Nov;12(3):189-98.

17 Lubben JE. Assessing social networks among elderly populations. Fam \& Community Health. 1988;11(3): 42-52.

18 Chang Q, Sha F, Chan CH, Yip PS. Validation of an abbreviated version of the Lubben Social Network Scale ("LSNS-6") and its associations with suicidality among older adults in China. PLoS One. 2018 Aug; 13(8):e0201612.

19 Zhang Z. Gender differentials in cognitive impairment and decline of the oldest old in China. J Gerontol B Psychol Sci Soc Sci. 2006 Mar;61(2):S107-15.

20 Steere HK, Quach L, Grande L, Bean JF. Evaluating the influence of social engagement on cognitive impairment and mobility outcomes within the Boston RISE cohort study. Am J Phys Med Rehabil. 2019 Aug;98(8):685-91.

21 Fratiglioni L, Wang HX, Ericsson K, Maytan M, Winblad B. Influence of social network on occurrence of dementia: a community-based longitudinal study. Lancet. 2000 Apr;355(9212):1315-9.

22 Haan MN. Can social engagement prevent cognitive decline in old age? Ann Intern Med. 1999 Aug;131(3): $220-1$.

23 Scott J, Carrington P. The SAGE handbook of social network analysis. London: SAGE; 2011.

24 Fabrigoule C, Letenneur L, Dartigues JF, Zarrouk M, Commenges D, Barberger-Gateau P. Social and leisure activities and risk of dementia: a prospective longitudinal study. J Am Geriatr Soc. 1995 May;43(5):485-90.

25 Verghese J, Lipton RB, Katz MJ, Hall CB, Derby CA, Kuslansky G, et al. Leisure activities and the risk of dementia in the elderly. N Engl J Med. 2003 Jun;348(25):2508-16.

26 Zheng W, Xiang YQ, Ungvari GS, Chiu HF, Ning YP, Yu X, et al. Tai chi for mild cognitive impairment: a systematic review. Psychogeriatrics. 2017 Nov;17(6):514-6.

27 Sungkarat S, Boripuntakul S, Chattipakorn N, Watcharasaksilp K, Lord SR. Effects of Tai Chi on Cognition and Fall Risk in Older Adults with Mild Cognitive Impairment: A Randomized Controlled Trial. J Am Geriatr Soc. 2017 Apr;65(4):721-7.

28 Ball K, Berch DB, Helmers KF, Jobe JB, Leveck MD, Marsiske M, et al.; Advanced Cognitive Training for Independent and Vital Elderly Study Group. Effects of cognitive training interventions with older adults: a randomized controlled trial. JAMA. 2002 Nov;288(18):2271-81.

29 Wilson RS, Bennett DA, Bienias JL, Aggarwal NT, Mendes De Leon CF, Morris MC, et al. Cognitive activity and incident AD in a population-based sample of older persons. Neurology. 2002 Dec;59(12):1910-4. 